\title{
Why Do Palliative Care Patients Request Deep Sedation at Home in France, and how Is Deep Sedation Conducted?
}

A Multicenter, Observational Case-control Study

EBERT Marie-Agnès, University of Montpellier, Palliative care department RICHARD Bruno, MD, University of Montpellier, Palliative care department

\section{INTRODUCTION}

New French Law on the End of Life (Leonetti-Claeys) in 2016 has permitted new rights for palliative care patients :

$\longrightarrow$ the right to decide better health way for themselves : right to autonomy

$\longrightarrow$ the right to decide to have a "deep and continuous sedation until death" is allowed under 3 circumstances
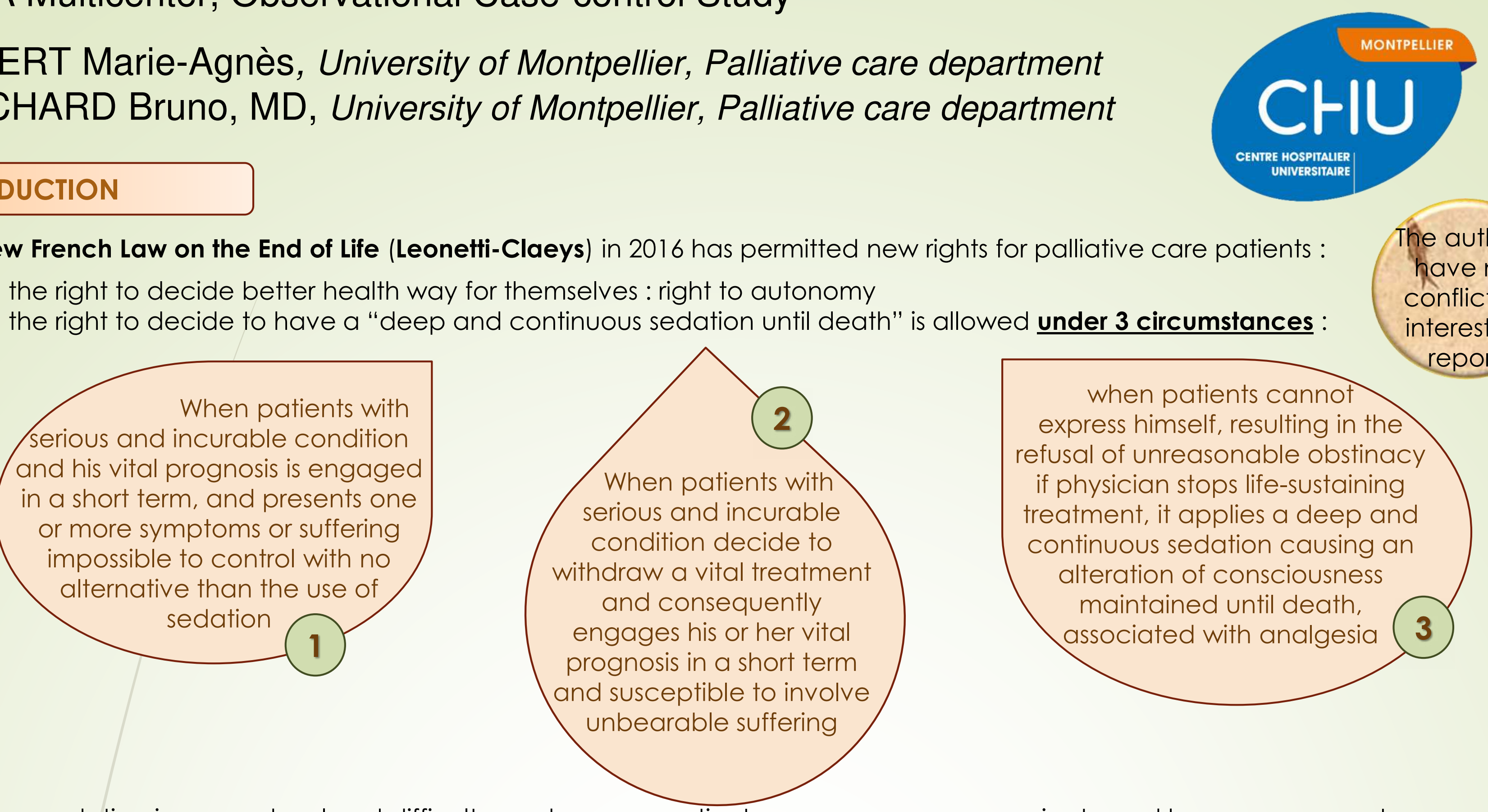

when patients cannot

express himself, resulting in the refusal of unreasonable obstinacy

if physician stops life-sustaining

treatment, it applies a deep and continuous sedation causing an

alteration of consciousness

maintained until death

associated with analgesia
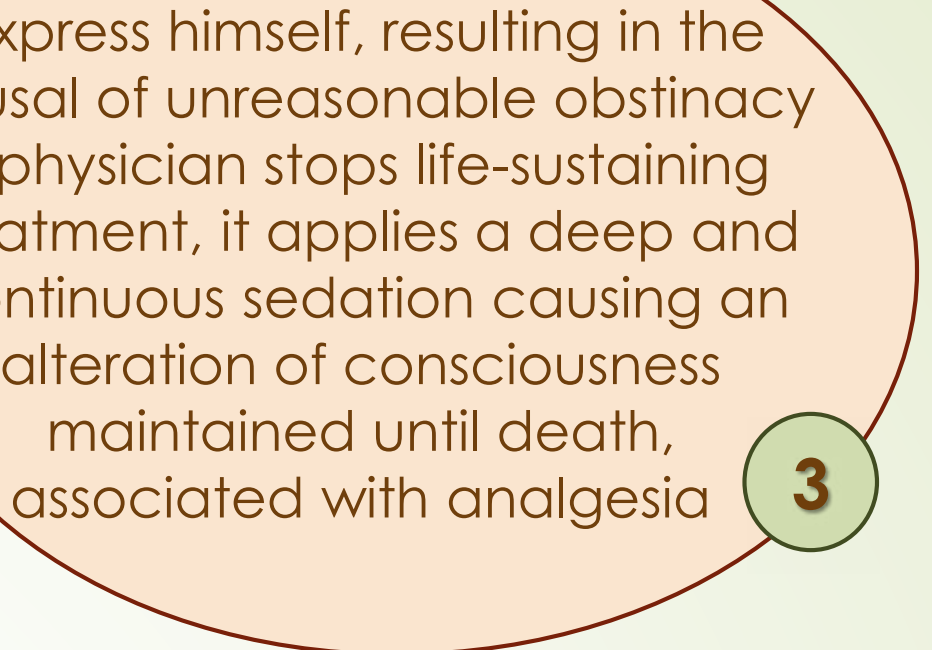

Deep sedation is a paradoxal and difficult care because patients can no more communicate and have no more autonomy. It can be stressful for doctors, nurses and caregivers who can feel deeply isolated especially at home.

The main aim of this study was to determine reasons of sedation requests to further anticipate them and maybe avoid them. The second aim was to determine how deep sedations were conducted at home.

\section{METHODS}

This multicenter observational case control study in 2018 lasted 3 months.

patients 18 y.o. or more

Decided to die at home

Life-threatening engaged in a short term

or a medium term

Received a deep sedation

Had a refractory suffering

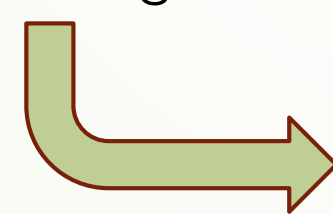

" refractory suffering " was defined " as any symptom that has been proposed and/or implemented with all available therapeutic and accompanying measures: without the relief expected by the patient; or causes unacceptable adverse effects; or their therapeutic effects are not likely to act within a period acceptable to the patient $॥$

All home palliative care teams and home care services on an area of $\mathbf{2 . 5}$ million people were associated to this study in order to include patients and controls and to collect data according to the questionnaire we elaborated. Sociodemographic data, spirituality, symptoms, prognosis, advance directives, surrogate decision-makers, medical evaluation,

20 consecutive patients were included over a period of 3 months. 11 had deep sedation and 9 were controls.

\begin{tabular}{|c|c|c|c|c|c|c|c|c|c|c|}
\hline Variables & N tot & $\mathbf{N}$ & $\mathbf{N}$ & $\%$ col & $\%$ lig & $\mathbf{N}$ & $\%$ col & \%lig & Test & P-Value \\
\hline Depression & 18 & 2 & 1 & 9,09 & 50,0 & 1 & 14,29 & 50 & Fisher & 0,1026 \\
\hline Unbearable suffering & 18 & 4 & 4 & 36,36 & 100,00 & 0 & 0,00 & 0,00 & Fisher & 0,1026 \\
\hline Nausea/vomiting & 20 & 1 & 1 & 9,09 & 100,00 & 0 & 0,00 & 0,00 & Fisher & 1,000 \\
\hline Generalised pain & 20 & 4 & 1 & 9,09 & 25,00 & 2 & 33,33 & 75 & Fisher & 0,2848 \\
\hline Anxiety & $\mathbf{2 0}$ & $\mathbf{1 1}$ & $\mathbf{9}$ & $\mathbf{8 1 , 8 2}$ & $\mathbf{8 1 , 8 2}$ & $\mathbf{2}$ & $\mathbf{2 2 , 2 2}$ & $\mathbf{1 8 , 1 7}$ & Fisher & $\mathbf{0 . 0 2 1 6}$ \\
\hline Insomnia & 20 & 2 & 1 & 9,09 & 50 & 1 & 11,11 & 50,00 & Fisher & 1.0000 \\
\hline Male & 20 & 14 & 9 & 81,82 & 64,29 & 5 & 55,56 & 35,71 & Fisher & 0,3359 \\
\hline
\end{tabular}

Most common indications for palliative sedation Delirium and dyspnea ${ }^{12}$ Sedated patients were younger ${ }^{12}$ Male ${ }^{12}$

Drugs used for palliative sedation

- Midazolam or Levomepromazine ${ }^{2}$

5 deep sedations were started according to advance care directives. Deep sedation was conducted with Midazolam in $\mathbf{9 0 , 9 \%}$ of patients, Clorazepate in $\mathbf{1 8 , 2 \%}$ and both in $\mathbf{9 , 1 \%}$. Titration was used in 5 of 11 patients.

\section{CONCLUSION}

Therefore home medical teams would focus on that symptom in order to better accompany and treat palliative care patients who want to die at home.

Could GPs reduce sedation number with screening and early treatment for anxiety? 